\title{
Synthesis of Novel Thiazolyl Hydrazine Derivatives and Their Antifungal Activity
}

\author{
Jianjun Zhu, Yazhen Chen, Fen Su, and Peiyi Wang (iD \\ State Key Laboratory Breeding Base of Green Pesticide and Agricultural Bioengineering, \\ Key Laboratory of Green Pesticide and Agricultural Bioengineering, Ministry of Education, \\ Center for R \& D of Fine Chemicals of Guizhou University, Guiyang 550025, China \\ Correspondence should be addressed to Peiyi Wang; pywang888@126.com
}

Received 12 June 2021; Revised 11 August 2021; Accepted 18 August 2021; Published 26 August 2021

Academic Editor: Vinod Kumar Tiwari

Copyright (c) 2021 Jianjun Zhu et al. This is an open access article distributed under the Creative Commons Attribution License, which permits unrestricted use, distribution, and reproduction in any medium, provided the original work is properly cited.

\begin{abstract}
A series of novel thiazolyl hydrazine derivatives 3a-3o were synthesized and evaluated for their in vitro antifungal activity against six phytopathogenic strains, namely, Botryosphaeria dothidea (B. d.), Gibberella sanbinetti (G. s.), Fusarium oxysporum (F. o.), Thanatephorus cucumeris (T. c.), Sclerotinia sclerotiorum (S. s.), and Verticillium dahliae (V. d.), by the classical mycelial growth rate method. Biological assessment results showed that most of these target compounds showed good antifungal activity toward tested strains. Especially, compound $\mathbf{3 1}$ showed excellent antifungal activities against $B . d$. and $G$. $s$. with relatively lower EC $\mathrm{E}_{50}$ values of 0.59 and $0.69 \mu \mathrm{g} / \mathrm{mL}$, respectively, which were extremely superior to those of commercial fungicides fluopyram, boscalid, and hymexazol and were comparable to those of carbendazim. Given the excellent bioactivity of designed compounds, this kind of thiazolyl hydrazine framework can provide a suitable point for exploring highly efficient antifungal agents.
\end{abstract}

\section{Introduction}

Fungal infection is a serious disease that affects the appearance, yield, and quality of various plants and fruits including wheat, potatoes, rape, maize, soybeans, apple, peppers, and cucumbers, thereby attracting considerable attention in recent years [1-7]. Pesticides that are capable of attacking and annihilating intractable phytopathogenic fungi play an important role in safeguarding our crops [8]. However, due to the frequent and excessive usage of traditional fungicides, drug-resistant pathogens gradually increase year after year [9]. Additionally, plants do not have the same immune system as animals; therefore, it is urgent to develop new eco-friendly pesticides with high bioactivity and selectivity to ensure the quality and yield of agricultural products.

Recently, thiazole derivatives have been strongly highlighted in consideration of their broad-spectrum pharmaceutical and pesticidal activities, such as insecticidal, herbicidal, antiviral, bactericidal, antimalarial, anti-cancer, antioxidant, and anti-tubercular functions [10-16]. Figure 1 illustrates some representative drugs containing the versatile thiazole scaffold including octhilinone, etridiazole $\left(\mathrm{EC}_{50}=0.006 \mu \mathrm{g} / \mathrm{mL}\right.$ against Phytophthora nicotianae $)$, amicarthiazol $\left(\mathrm{EC}_{50}=0.574 \mu \mathrm{g} / \mathrm{mL}\right.$ against Xanthomonas oryzae pv. oryzae, serving as bactericidal agents), and ethaboxam $\left(\mathrm{EC}_{50}=0.006 \mu \mathrm{g} / \mathrm{mL}\right.$ against Phytophthora nicotianae, serving as a fungicidal agent) [17-20]. On the other hand, molecular structures containing the hydrazide skeleton exhibit various biological activities such as antifungal, insecticidal, and weeding [21-23]. For instance, the correlative insecticides ANS-118 $\left(\mathrm{LC}_{50}=6.3 \mu \mathrm{g} / \mathrm{mL}\right.$ against beet armyworm) and RH-5849 $\left(\mathrm{EC}_{50}=0.095 \mu \mathrm{g} / \mathrm{mL}\right.$ against Daphnia magna), plant growth regulator daminozide, and bactericide benquinox were commercialized successively [24-27]. Therefore, thiazole and hydrazide scaffolds were considered as important skeletons in the fabrication of active molecules. In our previous works, we found that $1,3,4-$ oxadiazole hydrazide/sulfone/thioether derivatives showed good biological activity against plant microbial diseases [28-30]. In particular, 1,3,4-oxadiazole hydrazide derivatives performed a relatively high bioactivity against 
phytopathogenic oomycetes and fungi [30]. Encouraged by those studies, herein, a series of novel thiazole hydrazide derivatives were synthesized to continue the exploration of superior bioactive substrates. All the target compounds were screened for the in vitro activities against phytopathogenic fungi, including Botryosphaeria dothidea (B. d.), Gibberella sanbinetti (G. s.), Fusarium oxysporum (F. o.), Thanatephorus cucumeris (T. c.), Sclerotinia sclerotiorum (S. s.), and Verticillium dahliae ( $V$. d.).

\section{Materials and Methods}

2.1. Instrument and Chemicals. The NMR spectra of the synthesized compounds were measured by Bruker BiospinAG-400 apparatus (Bruker Optics, Switzerland). DMSO and TMS were used as the solvent and internal standard, respectively. All chemicals were purchased from Energy Chemical and used without further purification. All solvents meet the standard of analytical purity. The reaction process was detected by TLC.

2.2. General Procedures for Preparing Intermediate 1. Different substituted thiobenzamide $(0.01 \mathrm{~mol})$, ethyl 2chloro-3-oxobutanoate $(0.012 \mathrm{~mol})$, and ethanol $(50 \mathrm{~mL})$ were added. After reacting for $3-4 \mathrm{~h}$ at $100^{\circ} \mathrm{C}$, the organic layer was dried, and then $100 \mathrm{~mL}$ ethyl acetate was added. The organic layer was washed by water, brine, dried with sodium sulfate, and filtered, followed by the removal of the solvent under vacuum. Intermediate $\mathbf{1}$ was obtained by using column chromatography with the elution solvent of petroleum ether/ethyl acetate $=30 / 1$.

2.3. General Procedures for Preparing Intermediates $\mathbf{2 a - 2 b}$. Intermediate $1(0.01 \mathrm{~mol})$ and sodium hydroxide solution $(0.03 \mathrm{~mol})$ were added in methanol $(30 \mathrm{~mL})$. After reacting for $2-4 \mathrm{~h}$ at $55^{\circ} \mathrm{C}$, methanol was removed by distillation under reduced pressure. Then, the concentrated hydrochloric acid was incrementally added until the $\mathrm{pH}$ of the system was 2-3. Finally, intermediates $\mathbf{2} \mathbf{a}-\mathbf{2} \mathbf{b}$ were obtained by filtration and desiccation. 2-(4-Chlorophenyl)-4-methylthiazole-5-carboxylic acid (2a): ${ }^{1} \mathrm{H}$ NMR $\left(500 \mathrm{MHz}, \mathrm{DMSO}-d_{6}\right) \delta 8.00-7.87$ (m, $2 \mathrm{H}$, phenyl-H), 7.57-7.47 (m, $2 \mathrm{H}$, phenyl-H), $2.62(\mathrm{~s}, 3 \mathrm{H}$, $\left.-\mathrm{CH}_{3}\right) ;{ }^{13} \mathrm{C}$ NMR $\left(101 \mathrm{MHz}\right.$, DMSO- $\left.d_{6}\right) \delta 167.4,163.2,160.1$, $136.3, \quad 129.8, \quad 128.6, \quad 123.7, \quad 17.5$. 4-Methyl-2-(4-(trifluoromethyl)phenyl)thiazole-5-carboxylic acid (2b): ${ }^{1} \mathrm{H}$ NMR $\left(400 \mathrm{MHz}\right.$, DMSO- $\left.d_{6}\right) \delta 8.17(\mathrm{~d}, J=8.1 \mathrm{~Hz}, 2 \mathrm{H}$, phenyl$\mathrm{H}), 7.86(\mathrm{~d}, J=8.3 \mathrm{~Hz}, 2 \mathrm{H}$, phenyl- $\mathrm{H}), 2.69\left(\mathrm{~s}, 3 \mathrm{H},-\mathrm{CH}_{3}\right) ;{ }^{13} \mathrm{C}$ NMR $\left(101 \mathrm{MHz}, \mathrm{DMSO}-d_{6}\right) \delta 166.8,163.2,160.2,136.2,131.2$ $\left(\mathrm{d},{ }^{2} J_{\mathrm{C}-\mathrm{F}}=32.1 \mathrm{~Hz}\right), 128.3,126.7\left(\mathrm{dd},{ }^{3} J_{\mathrm{C}-\mathrm{F}}=7.3,3.5 \mathrm{~Hz}\right), 125.3$ $\left(\mathrm{dd},{ }^{1} J_{\mathrm{C}-\mathrm{F}}=289.5,191.6 \mathrm{~Hz}\right), 124.7,17.5 ;{ }^{19} \mathrm{~F}$ NMR $(377 \mathrm{MHz}$, DMSO- $\left.d_{6}\right) \delta-61.4$.

2.4. General Procedures for Preparing the Target Compounds $\mathbf{3 a}$-3o. Intermediates $\mathbf{2 a}$ or $\mathbf{2 b}(0.01 \mathrm{~mol})$, 1-ethyl-3-(3dimethyllaminopropyl) carbodiimide hydrochloride (EDCI, $0.02 \mathrm{~mol}), 1$-hydroxybenzotriazole $(0.012 \mathrm{~mol})$, and triethylamine $(0.012 \mathrm{~mol})$ were added in $10 \mathrm{~mL} \mathrm{CH} \mathrm{Cl}_{2}$.
After the mixture was completely dissolved, different substituted phenylhydrazines were added. Then, the reaction was stirred for $4-5 \mathrm{~h}$ at $25^{\circ} \mathrm{C}$. After that, $50 \mathrm{~mL}$ $\mathrm{CH}_{2} \mathrm{Cl}_{2}$ was added into the mixture. And the organic layer was washed by water, brine, dried with sodium sulfate, and filtered, followed by the removal of the solvent under vacuum. Finally, target compounds $\mathbf{3 a - 3 o}$ were obtained by recrystallization in ethanol. 2-(4-Chlorophenyl)-4methyl- $\mathrm{N}^{\prime}$-phenylthiazole-5-carbohydrazide (3a): a yellow solid, m. p. $185.6-186.7^{\circ} \mathrm{C}$, yield $19.7 \% ;{ }^{1} \mathrm{H}$ NMR $(400 \mathrm{MHz}$, DMSO- $\left.d_{6}\right) \delta 10.22(\mathrm{~s}, 1 \mathrm{H},-\mathrm{CONH}-), 8.02(\mathrm{~s}, 1 \mathrm{H},-\mathrm{NH}-)$, $7.98(\mathrm{~d}, J=8.6 \mathrm{~Hz}, 2 \mathrm{H}$, phenyl-H), $7.59(\mathrm{~d}, J=8.5 \mathrm{~Hz}, 2 \mathrm{H}$, phenyl-H), $7.18(\mathrm{t}, J=7.8 \mathrm{~Hz}, 2 \mathrm{H}$, phenyl-H), 6.79 (d, $J=7.9 \mathrm{~Hz}, 2 \mathrm{H}$, phenyl- $\mathrm{H}), 6.74(\mathrm{t}, J=7.3 \mathrm{~Hz}, 1 \mathrm{H}$, phenyl$\mathrm{H}), 2.67\left(\mathrm{~s}, 3 \mathrm{H},-\mathrm{CH}_{3}\right) ;{ }^{13} \mathrm{C}$ NMR $\left(101 \mathrm{MHz}\right.$, DMSO- $\left.d_{6}\right) \delta$ 165.6, 161.8, 156.6, 149.5, 136.1, 131.6, 129.9, 129.3, 128.5, 124.7, 119.3, 112.8, 17.6; HRMS (ESI) $[\mathrm{M}-\mathrm{H}]^{-}$calcd for $\mathrm{C}_{17} \mathrm{H}_{15} \mathrm{ON}_{3} \mathrm{ClS}: 344.0619$, found: 344.0619 .

2.5. 2-(4-Chlorophenyl)-N'-(2-fluorophenyl)-4-methylthiaz ole-5-carbohydrazide (3b). A yellow solid, m. p. $163.2-164.3^{\circ} \mathrm{C}$, yield $25.7 \%$; ${ }^{1} \mathrm{H}$ NMR $\left(400 \mathrm{MHz}, \mathrm{DMSO}-d_{6}\right)$ $\delta 10.29$ (s, 1H, -CONH-), 7.98 (d, $J=8.6 \mathrm{~Hz}, 2 \mathrm{H}$, phenyl-H), 7.95 (s, 1H, -NH-), 7.59 (d, J=8.5 Hz, 2H, phenyl-H), 7.11 $(\mathrm{d}, J=12.0,8.1,0.9 \mathrm{~Hz}, 1 \mathrm{H}$, phenyl-H), $7.03(\mathrm{t}, J=7.7 \mathrm{~Hz}$, $1 \mathrm{H}$, phenyl-H), 6.84 (dd, $J=12.2,4.5 \mathrm{~Hz}, 1 \mathrm{H}$, phenyl- $\mathrm{H})$, $6.76(\mathrm{~d}, J=9.0,6.4,1.4 \mathrm{~Hz}, 1 \mathrm{H}$, phenyl-H), $2.67(\mathrm{~s}, 3 \mathrm{H}$, $\left.-\mathrm{CH}_{3}\right) ;{ }^{13} \mathrm{C}$ NMR $\left(101 \mathrm{MHz}, \mathrm{DMSO}-d_{6}\right) \delta 165.7,161.9$, $156.9,150.7\left(\mathrm{~d},{ }^{1} J_{\mathrm{C}-\mathrm{F}}=239.0 \mathrm{~Hz}\right), 137.1\left(\mathrm{~d},{ }^{2} J_{\mathrm{C}-\mathrm{F}}=10.6 \mathrm{~Hz}\right)$, $136.1,131.6,129.9,128.5,125.1\left(\mathrm{~d},{ }^{4} J_{\mathrm{C}-\mathrm{F}}=2.8 \mathrm{~Hz}\right), 124.5$, $119.5\left(\mathrm{~d},{ }^{3} J_{\mathrm{C}-\mathrm{F}}=6.8 \mathrm{~Hz}\right), 115.5\left(\mathrm{~d},{ }^{2} J_{\mathrm{C}-\mathrm{F}}=17.9 \mathrm{~Hz}\right), 114.2(\mathrm{~d}$, $\left.{ }^{3} J_{\mathrm{C}-\mathrm{F}}=3.0 \mathrm{~Hz}\right), 17.64 ;{ }^{19} \mathrm{~F}$ NMR $\left(377 \mathrm{MHz}, \mathrm{DMSO}-d_{6}\right) \delta$ -132.9 ; HRMS (ESI) $[\mathrm{M}-\mathrm{H}]^{-}$calcd for $\mathrm{C}_{17} \mathrm{H}_{14} \mathrm{ON}_{3} \mathrm{ClS}$ : 362.0525, found: 362.0525 .

2.6. 2-(4-Chlorophenyl)-N'-(3-fluorophenyl)-4-methylthia zole-5-carbohydrazide (3c). A yellow solid, m. p. 195.9-196. $4^{\circ} \mathrm{C}$, yield 40.5\%; ${ }^{1} \mathrm{H}$ NMR (500 MHz, DMSO- $d_{6}$ ) $\delta 10.29(\mathrm{~s}, 1 \mathrm{H},-\mathrm{CONH}-), 8.35$ (s, 1H, -NH-), $7.98(\mathrm{t}$, $J=11.3 \mathrm{~Hz}, 2 \mathrm{H}$, phenyl-H), $7.58(\mathrm{dd}, J=22.7,8.6 \mathrm{~Hz}, 2 \mathrm{H}$, phenyl-H), $7.24-7.15(\mathrm{~m}, 1 \mathrm{H}$, phenyl-H), 6.64 (dd, $J=15.0$, $4.7 \mathrm{~Hz}, 1 \mathrm{H}$, phenyl-H), $6.62-6.55(\mathrm{~m}, 1 \mathrm{H}$, phenyl-H), 6.53 (d, $J=6.8 \mathrm{~Hz}, 1 \mathrm{H}$, phenyl-H), 6.50 (s, 1H, phenyl-H), 2.67 $\left(\mathrm{s}, 3 \mathrm{H},-\mathrm{CH}_{3}\right) ;{ }^{13} \mathrm{C} \mathrm{NMR}\left(101 \mathrm{MHz}, \mathrm{DMSO}-d_{6}\right) \delta 165.7$, $163.7\left(\mathrm{~d},{ }^{1} J_{\mathrm{C}-\mathrm{F}}=240.6 \mathrm{~Hz}\right), 161.8,157.0,151.7\left(\mathrm{~d},{ }^{3} J_{\mathrm{C}-}\right.$ $\mathrm{F}=10.1 \mathrm{~Hz}), 136.1,131.6,131.0\left(\mathrm{~d},{ }^{3} J_{\mathrm{C}-\mathrm{F}}=10.0 \mathrm{~Hz}\right), 129.9$, 128.6, 124.4, 108.7, $105.4\left(\mathrm{~d},{ }^{2} J_{\mathrm{C}-\mathrm{F}}=21.2 \mathrm{~Hz}\right), 99.2\left(\mathrm{~d},{ }^{2} J_{\mathrm{C}-}\right.$ $\mathrm{F}=25.7 \mathrm{~Hz}), \quad 17.7 ;{ }^{19} \mathrm{~F}$ NMR $\left(377 \mathrm{MHz}, \quad \mathrm{DMSO}-d_{6}\right) \quad \delta$ -133.0; HRMS (ESI) $[\mathrm{M}-\mathrm{H}]^{-}$calcd for $\mathrm{C}_{17} \mathrm{H}_{12} \mathrm{ON}_{3} \mathrm{ClFS}$ : 360.0382, found: 360.0368 .

2.7. 2-(4-Chlorophenyl)-N'-(4-fluorophenyl)-4-methylthiazo le-5-carbohydrazide (3d). A yellow solid, m. p. 174.1-175.6 ${ }^{\circ}$, yield 29.4\%; ${ }^{1} \mathrm{H}$ NMR (400 MHz, DMSO$\left.d_{6}\right) \delta 10.24(\mathrm{~s}, 1 \mathrm{H},-\mathrm{CONH}-), 8.00(\mathrm{~s}, 1 \mathrm{H},-\mathrm{NH}-), 7.98(\mathrm{~d}$, $J=8.6 \mathrm{~Hz}, 2 \mathrm{H}$, phenyl-H), 7.63-7.56 ( $\mathrm{m}, 2 \mathrm{H}$, phenyl- $\mathrm{H})$, $7.02(\mathrm{t}, J=8.8 \mathrm{~Hz}, 2 \mathrm{H}$, phenyl-H), 6.82-6.77 (m, $2 \mathrm{H}$, 

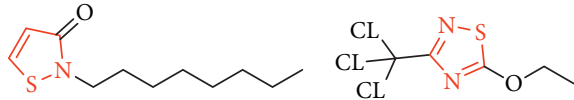

Octhilinone

(Bactericide)

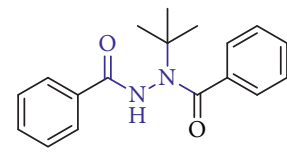

RH-5849

(Insecticide)
Etridiazole

(Bactericide)<smiles>Cc1nc(N)sc1C(=O)Nc1ccccc1</smiles>
Amicarthiazol
(Bactericide)<smiles>CCNc1nc(CC)c(C(=O)NC(C#N)c2cccs2)s1</smiles>

Ethaboxam (Fungicide)<smiles>Cc1cc(C)cc(C(=O)N(NC(=O)c2ccc3c(c2C)CCCO3)C(C)(C)C)c1</smiles>

Benquinox (Fungicide)

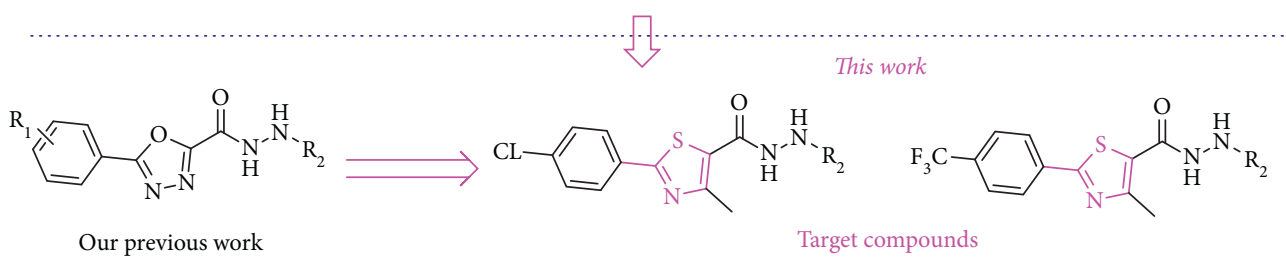

FiguRE 1: Structure of few selected thiazolyl or carbohydrazide-containing drug candidates and the related design strategy for the target molecules.

phenyl-H), $2.66\left(\mathrm{~s}, 3 \mathrm{H},-\mathrm{CH}_{3}\right) ;{ }^{13} \mathrm{C} \mathrm{NMR}(101 \mathrm{MHz}$, $\left.\mathrm{DMSO}-d_{6}\right) \quad \delta \quad 165.6,161.8,157.6,156.5 \quad\left(\mathrm{~d},{ }^{1} J_{\mathrm{C}-}\right.$ $\mathrm{F}=233.3 \mathrm{~Hz}), 146.1,136.1,131.6,129.9\left(\mathrm{~d},{ }^{3} J_{\mathrm{C}-\mathrm{F}}=11.2 \mathrm{~Hz}\right)$, $128.5,124.6,115.8\left(\mathrm{~d},{ }^{2} J_{\mathrm{C}-\mathrm{F}}=22.2 \mathrm{~Hz}\right), 114.0\left(\mathrm{~d},{ }^{4} J_{\mathrm{C}-}\right.$ $\mathrm{F}=7.6 \mathrm{~Hz}), 17.6 ;{ }^{19} \mathrm{~F}$ NMR $\left(377 \mathrm{MHz}, \mathrm{DMSO}-d_{6}\right) \delta-126.2$; HRMS (ESI) $[\mathrm{M}-\mathrm{H}]^{+}$calcd for $\mathrm{C}_{17} \mathrm{H}_{12} \mathrm{ON}_{3} \mathrm{ClFS}$ : 360.0368, found: 360.0382 .

2.8. $N^{\prime}$-(4-Bromophenyl)-2-(4-chlorophenyl)-4-methylthiazo le-5-carbohydrazide (3e). A yellow solid, m. p. 237.7-238.2 ${ }^{\circ} \mathrm{C}$, yield $31.9 \%$; ${ }^{1} \mathrm{H}$ NMR (400 MHz, DMSO$\left.d_{6}\right) \delta 10.26(\mathrm{~s}, 1 \mathrm{H},-\mathrm{CONH}-), 8.23(\mathrm{~s}, 1 \mathrm{H},-\mathrm{NH}-), 7.98(\mathrm{~d}$, $J=8.5 \mathrm{~Hz}, 2 \mathrm{H}$, phenyl-H), 7.59 (d, $J=8.5 \mathrm{~Hz}, 2 \mathrm{H}$, phenyl$\mathrm{H}), 7.33(\mathrm{~d}, J=8.7 \mathrm{~Hz}, 2 \mathrm{H}$, phenyl-H), $6.74(\mathrm{~d}, J=8.8 \mathrm{~Hz}$, $2 \mathrm{H}$, phenyl-H), $2.66\left(\mathrm{~s}, 3 \mathrm{H},-\mathrm{CH}_{3}\right) ;{ }^{13} \mathrm{C} \mathrm{NMR}(101 \mathrm{MHz}$, DMSO- $\left.d_{6}\right) \delta 165.7,161.8,157.0,148.9,136.1,131.9,131.6$, $129.9,128.5,124.4,114.8,110.1,17.7 ;{ }^{19} \mathrm{~F}$ NMR $(377 \mathrm{MHz}$, DMSO- $\left.d_{6}\right) \quad \delta-61.4$; HRMS (ESI) $[\mathrm{M}-\mathrm{H}]^{-}$calcd for $\mathrm{C}_{17} \mathrm{H}_{12} \mathrm{ON}_{3} \mathrm{BrClS}$ : 419.9567, found: 419.9587 .

2.9. 2-(4-Chlorophenyl)-4-methyl-N'-(4-(trifluoromethyl)phe nyl)thiazole-5-carbohydrazide (3f). A white solid, m. p. 248.3-249.5 ${ }^{\circ} \mathrm{C}$, yield 28.3\%; ${ }^{1} \mathrm{H}$ NMR (400 MHz, DMSO$\left.d_{6}\right) \delta 10.37$ (s, 1H, -CONH-), 8.67 (s, 1H, -NH-), 8.00 (d, $J=8.5 \mathrm{~Hz}, 2 \mathrm{H}$, phenyl-H), $7.61(\mathrm{~d}, J=8.5 \mathrm{~Hz}, 2 \mathrm{H}$, phenyl$\mathrm{H}), 7.52(\mathrm{~d}, J=8.5 \mathrm{~Hz}, 2 \mathrm{H}$, phenyl-H), $6.90(\mathrm{~d}, J=8.5 \mathrm{~Hz}$, $2 \mathrm{H}$, phenyl-H), $2.68\left(\mathrm{~s}, 3 \mathrm{H},-\mathrm{CH}_{3}\right) ;{ }^{13} \mathrm{C} \mathrm{NMR}(101 \mathrm{MHz}$, DMSO- $\left.d_{6}\right) \delta 165.8,161.8,157.2,152.6,136.2,131.6,123.0$, $128.6,126.8\left(\mathrm{dd},{ }^{3} J_{\mathrm{C}-\mathrm{F}}=11.9,2.8 \mathrm{~Hz}\right), 125.5\left(\mathrm{dd},{ }^{1} J_{\mathrm{C}-}\right.$ $\mathrm{F}=257.55 \mathrm{~Hz}), 119.1,119.0\left(\mathrm{dd},{ }^{2} J_{\mathrm{C}-\mathrm{F}}=63.2,31.7 \mathrm{~Hz}\right), 112.1$, $17.7 ;{ }^{19} \mathrm{~F}$ NMR $\left(377 \mathrm{MHz}, \mathrm{DMSO}-d_{6}\right) \delta-59.4$; HRMS (ESI) $[\mathrm{M}-\mathrm{H}]^{-}$calcd for $\mathrm{C}_{18} \mathrm{H}_{14} \mathrm{ON}_{3} \mathrm{ClF}_{3} \mathrm{~S}$ : 412.0493, found: 412.0493 .
2.10. $N^{\prime}$-(3-Chlorophenyl)-2-(4-chlorophenyl)-4-methylthiaz ole-5-carbohydrazide (3g). A white solid, $\mathrm{m}$. p. 197.1-199. $3^{\circ} \mathrm{C}$, yield 63.7\%; ${ }^{1} \mathrm{H}$ NMR (400 MHz, DMSO- $\left.d_{6}\right)$ $\delta 10.28$ (s, 1H, -CONH-), 8.34 (s, 1H, -NH-), 7.98 (d, $J=8.5 \mathrm{~Hz}, 2 \mathrm{H}$, phenyl-H), 7.59 (d, $J=8.5 \mathrm{~Hz}, 2 \mathrm{H}$, phenyl-H), $7.19(\mathrm{t}, J=8.0 \mathrm{~Hz}, 1 \mathrm{H}$, phenyl-H), 6.77 (s, 1H, phenyl-H), $6.75(\mathrm{~s}, 1 \mathrm{H}$, phenyl-H), $6.73(\mathrm{~s}, 1 \mathrm{H}$, phenyl-H), $2.67(\mathrm{~s}, 3 \mathrm{H}$, $\left.-\mathrm{CH}_{3}\right) ;{ }^{13} \mathrm{C}$ NMR $\left(101 \mathrm{MHz}\right.$, DMSO- $\left.d_{6}\right) \delta 165.7,161.8,157.0$, 151.1, 136.1, 134.0, 131.6, 131.0, 129.9, 128.6, 124.3, 118.7, 112.0, 111.4, 17.7; HRMS (ESI) [M-H $]^{-}$calcd for $\mathrm{C}_{17} \mathrm{H}_{14} \mathrm{ON}_{3} \mathrm{Cl}_{2} \mathrm{~S}$ : 378.0225, found: 378.0227.

2.11. $N^{\prime}$-(4-Chlorophenyl)-2-(4-chlorophenyl)-4-methylthiaz ole-5-carbohydrazide (3h). A yellow solid, m. p. $164.5-165.6^{\circ} \mathrm{C}$, yield $13.7 \% ;{ }^{1} \mathrm{H}$ NMR $\left(400 \mathrm{MHz}, \mathrm{DMSO}-d_{6}\right)$ $\delta 10.26$ (s, 1H, -CONH-), 8.21 (s, 1H, -NH-), 7.97 (d, $J=8.5 \mathrm{~Hz}, 2 \mathrm{H}$, phenyl-H), 7.58 (d, $J=8.5 \mathrm{~Hz}, 2 \mathrm{H}$, phenyl-H), $7.21(\mathrm{~d}, J=8.7 \mathrm{~Hz}, 2 \mathrm{H}$, phenyl-H), $6.79(\mathrm{~d}, J=8.8 \mathrm{~Hz}, 2 \mathrm{H}$, phenyl-H), 2.66 (s, 3H, - $\left.\mathrm{CH}_{3}\right) ;{ }^{13} \mathrm{C}$ NMR (101 MHz, DMSO$\left.d_{6}\right) \delta 165.7,161.8,156.9,148.5,136.1,131.6,129.9,129.1$, 128.5, 124.4, 122.6, 114.3, 17.7; HRMS (ESI) $[\mathrm{M}-\mathrm{H}]^{-}$calcd for $\mathrm{C}_{17} \mathrm{H}_{12} \mathrm{ON}_{3} \mathrm{Cl}_{2} \mathrm{~S}$ : 376.0073 , found: 376.0086 .

2.12. 4-Methyl-N'-phenyl-2-(4-(trifluoromethyl)phenyl)thiazole-5-carbohydrazide (3i). A yellow solid, m. p. 182.6-183.3 ${ }^{\circ} \mathrm{C}$, yield $38.8 \% ;{ }^{1} \mathrm{H}$ NMR (400 MHz, DMSO- $\left.d_{6}\right) \delta$ 10.28 (s, 1H, -CONH-), 8.18 (d, J=8.1 Hz, 2H, phenyl-H), 8.03 (s, 1H, -NH-), 7.89 (d, J=8.3 Hz, 2H, phenyl-H), $7.18(\mathrm{t}$, $J=7.9 \mathrm{~Hz}, 2 \mathrm{H}$, phenyl-H), $6.80(\mathrm{~d}, J=7.8 \mathrm{~Hz}, 2 \mathrm{H}$, phenyl-H), $6.74\left(\mathrm{t}, J=7.3 \mathrm{~Hz}, 1 \mathrm{H}\right.$, phenyl-H), $2.69\left(\mathrm{~s}, 3 \mathrm{H},-\mathrm{CH}_{3}\right) ;{ }^{13} \mathrm{C} \mathrm{NMR}$ $\left(101 \mathrm{MHz}, \mathrm{DMSO}-d_{6}\right) \delta 165.0,161.7,156.8,149.5,136.3,129.6$, $129.3,127.6,126.8\left(\mathrm{dd},{ }^{2} J_{\mathrm{C}-\mathrm{F}}=9.8,5.9 \mathrm{~Hz}\right), 125.6,124.5\left(\mathrm{dd},{ }^{1} J_{\mathrm{C}-}\right.$ $\mathrm{F}=523.4,251.0 \mathrm{~Hz}), 119.3,112.8,17.6 .{ }^{19} \mathrm{~F} \mathrm{NMR}(377 \mathrm{MHz}$, DMSO- $\left.d_{6}\right) \quad \delta \quad-61.4$; HRMS (ESI) $[\mathrm{M}-\mathrm{H}]^{-}$calcd for $\mathrm{C}_{18} \mathrm{H}_{15} \mathrm{ON}_{3} \mathrm{~F}_{3} \mathrm{~S}$ : 378.0882 , found: 378.0875 . 
2.13. $\quad N^{\prime}$-(2-Fluorophenyl)-4-methyl-2-(4-(trifluoromethyl) phenyl)thiazole-5-carbohydrazide (3j). A white solid, m. p. $148.5-149.6^{\circ} \mathrm{C}$, yield $43.0 \% ;{ }^{1} \mathrm{H}$ NMR $\left(400 \mathrm{MHz}, \mathrm{DMSO}-d_{6}\right) \delta$ 10.35 (s, 1H, -CONH-), 8.18 (d, J=8.1 Hz, 2H, phenyl-H), 7.97 (s, 1H, -NH-), 7.89 (d, J=8.3 Hz, 2H, phenyl-H), 7.15 - 7.07 (m, 1H, phenyl-H), 7.03 (t, $J=7.7 \mathrm{~Hz}, 1 \mathrm{H}$, phenyl-H), $6.89-6.83(\mathrm{~m}, 1 \mathrm{H}$, phenyl-H), $6.79-6.72(\mathrm{~m}, 1 \mathrm{H}$, phenyl-H), 2.69 (s, 3H, $\left.-\mathrm{CH}_{3}\right) ;{ }^{13} \mathrm{C}$ NMR (101 MHz, DMSO- $\left.d_{6}\right) \delta 165.1$, 161.8, 157.1, $150.7 \quad\left(\mathrm{~d},{ }^{1} J_{\mathrm{C}-\mathrm{F}}=239.2 \mathrm{~Hz}\right), 137.0 \quad\left(\mathrm{~d},{ }^{2} J_{\mathrm{C}-}\right.$ $\left.\mathrm{F}_{\mathrm{F}}=10.6 \mathrm{~Hz}\right), 136.3,131.1\left(\mathrm{~d},{ }^{2} J_{\mathrm{C}-\mathrm{F}}=32.1 \mathrm{~Hz}\right), 127.7,126.8(\mathrm{dd}$, $\left.{ }^{3} J_{\mathrm{C}-\mathrm{F}}=10.3,6.4 \mathrm{~Hz}\right), 125.4,125.1\left(\mathrm{~d},{ }^{4} J_{\mathrm{C}-\mathrm{F}}=2.9 \mathrm{~Hz}\right), 124.4(\mathrm{q}$, $\left.{ }^{1} J_{\mathrm{C}-\mathrm{F}}=272.0 \mathrm{~Hz}\right), 119.6\left(\mathrm{~d},{ }^{1} J_{\mathrm{C}-\mathrm{F}}=6.7 \mathrm{~Hz}\right), 115.5\left(\mathrm{~d},{ }^{2} J_{\mathrm{C}-}\right.$ $\mathrm{F}=17.7 \mathrm{~Hz}), \quad 114.2 \quad\left(\mathrm{~d},{ }^{3} J_{\mathrm{C}-\mathrm{F}}=3.0 \mathrm{~Hz}\right), \quad 17.6 ;{ }^{19} \mathrm{~F} \quad \mathrm{NMR}$ $\left(377 \mathrm{MHz}, \mathrm{DMSO}-d_{6}\right) \delta-61.4,-132.9$; HRMS (ESI) $[\mathrm{M}-\mathrm{H}]^{+}$ calcd for $\mathrm{C}_{18} \mathrm{H}_{12} \mathrm{ON}_{3} \mathrm{~F}_{4} \mathrm{~S}$ : 394.0632, found: 394.0643 .

2.14. $\quad N^{\prime}$-(3-Fluorophenyl)-4-methyl-2-(4-(trifluoromethyl) phenyl)thiazole-5-carbohydrazide ( $3 \boldsymbol{k})$. A yellow solid, m. p. 167.4-168. $7^{\circ} \mathrm{C}$, yield $25.4 \%$; ${ }^{1} \mathrm{H}$ NMR $\left(400 \mathrm{MHz}, \mathrm{DMSO}-d_{6}\right)$ $\delta 10.34$ (s, 1H, -CONH-) 8.35 (s, 1H, -NH-), 8.18 (d, $J=8.1 \mathrm{~Hz}, 2 \mathrm{H}$, phenyl-H), $7.88(\mathrm{~d}, J=8.3 \mathrm{~Hz}, 2 \mathrm{H}$, phenyl-H), 7.20 (dd, $J=15.4,8.1 \mathrm{~Hz}, 1 \mathrm{H}$, phenyl-H), 6.65-6.61 (m, 1H, phenyl-H), $6.54(\mathrm{~s}, 1 \mathrm{H}$, phenyl-H), 6.52-6.48 (m, $1 \mathrm{H}$, phenyl-H), 2.69 (s, 3H, $\left.-\mathrm{CH}_{3}\right) ;{ }^{13} \mathrm{C}$ NMR (101 MHz, DMSO$\left.d_{6}\right) \delta 165.1,163.7\left(\mathrm{~d},{ }^{1} J_{\mathrm{C}-\mathrm{F}}=240.7 \mathrm{~Hz}\right), 161.7,157.2,151.7(\mathrm{~d}$, $\left.{ }^{3} J_{\mathrm{C}-\mathrm{F}}=10.3 \mathrm{~Hz}\right), 136.3,131.3\left(\mathrm{~d},{ }^{3} J_{\mathrm{C}-\mathrm{F}}=10.3 \mathrm{~Hz}\right), 131.0(\mathrm{~d}$, $\left.{ }^{2} J_{\mathrm{C}-\mathrm{F}}=10.4 \mathrm{~Hz}\right), 127.6,126.8\left(\mathrm{dd},{ }^{3} J_{\mathrm{C}-\mathrm{F}}=9.0,5.6 \mathrm{~Hz}\right), 125.3$, $124.4\left(\mathrm{q},{ }^{1} J_{\mathrm{C}-\mathrm{F}}=272.4 \mathrm{~Hz}\right), 108.7\left(\mathrm{~d},{ }^{4} J_{\mathrm{C}-\mathrm{F}}=0.9 \mathrm{~Hz}\right), 105.4(\mathrm{~d}$, $\left.{ }^{2} J_{\mathrm{C}-\mathrm{F}}=21.3 \mathrm{~Hz}\right), 99.3\left(\mathrm{~d},{ }^{2} J_{\mathrm{C}-\mathrm{F}}=25.7 \mathrm{~Hz}\right), 17.6 ;{ }^{19} \mathrm{~F} \mathrm{NMR}$ $\left(377 \mathrm{MHz}, \mathrm{DMSO}-d_{6}\right) \delta-61.4,-113.1$; HRMS (ESI) $[\mathrm{M}-\mathrm{H}]^{+}$ calcd for $\mathrm{C}_{18} \mathrm{H}_{12} \mathrm{ON}_{3} \mathrm{~F}_{4} \mathrm{~S}$ : 394.0632, found: 394.0639 .

2.15. $\quad N^{\prime}$-(4-Fluorophenyl)-4-methyl-2-(4-(trifluoromethyl) phenyl)thiazole-5-carbohydrazide (3l). A yellow solid, m. p. 186.3-187.9 ${ }^{\circ} \mathrm{C}$, yield $42.7 \%{ }^{1} \mathrm{H}$ NMR $\left(400 \mathrm{MHz}, \mathrm{DMSO}-d_{6}\right) \delta$ 10.31 (s, 1H, -CONH-), 8.17 (d, $J=8.1 \mathrm{~Hz}, 2 \mathrm{H}$, phenyl-H), 8.02 (s, $1 \mathrm{H},-\mathrm{NH}-), 7.88$ (d, J=8.3 Hz, 2H, phenyl-H), 7.03 (t, $J=8.9 \mathrm{~Hz}, 2 \mathrm{H}$, phenyl-H), 6.81 (dd, $J=9.0,4.6 \mathrm{~Hz}, 2 \mathrm{H}$, phenyl$\mathrm{H}), 2.68\left(\mathrm{~s}, 3 \mathrm{H},-\mathrm{CH}_{3}\right) ;{ }^{13} \mathrm{C} \mathrm{NMR}\left(101 \mathrm{MHz}, \mathrm{DMSO}-d_{6}\right) \delta$ $165.0,161.7,156.9,156.5\left(\mathrm{~d},{ }^{1} J_{\mathrm{C}-\mathrm{F}}=233.7 \mathrm{~Hz}\right), 146.0\left(\mathrm{~d},{ }^{4} J_{\mathrm{C}-}\right.$ $\mathrm{F}=0.9 \mathrm{~Hz}), 136.3,128.4,127.5\left(\mathrm{~d},{ }^{2} J_{\mathrm{C}-\mathrm{F}}=20.2 \mathrm{~Hz}\right), 126.8(\mathrm{dd}$, $\left.{ }^{3} J_{\mathrm{C}-\mathrm{F}}=8.2,4.9 \mathrm{~Hz}\right), 125.5,124.4\left(\mathrm{q},{ }^{1} J_{\mathrm{C}-\mathrm{F}}=272.5 \mathrm{~Hz}\right), 115.8(\mathrm{~d}$, $\left.{ }^{2} J_{\mathrm{C}-\mathrm{F}}=22.3 \mathrm{~Hz}\right), \quad 114.0 \quad\left(\mathrm{~d},{ }^{3} J_{\mathrm{C}-\mathrm{F}}=7.6 \mathrm{~Hz}\right), 17.6 ;{ }^{19} \mathrm{~F} \quad \mathrm{NMR}$ $\left(377 \mathrm{MHz}, \mathrm{DMSO}-d_{6}\right) \delta-61.4,-126.1$; HRMS (ESI) $[\mathrm{M}-\mathrm{H}]^{-}$ calcd for $\mathrm{C}_{18} \mathrm{H}_{12} \mathrm{ON}_{3} \mathrm{~F}_{4} \mathrm{~S}$ : 394.0632, found: 394.0640 .

2.16. $\quad N^{\prime}$-(4-Bromophenyl)-4-methyl-2-(4-(trifluoromethyl) phenyl)thiazole-5-carbohydrazide (3m). A yellow solid, m. p. 214.0-215.2 ${ }^{\circ} \mathrm{C}$, yield 50.3\%; ${ }^{1} \mathrm{H}$ NMR (400 MHz, DMSO- $\left.d_{6}\right) \delta$ 10.32 (s, 1H, -CONH-), 8.25 (s, 1H, -NH-), 8.18 (d, J=8.1 Hz, 2H, phenyl-H), 7.89 (d, $J=8.3 \mathrm{~Hz}, 2 \mathrm{H}$, phenyl-H), 7.33 (d, $J=8.8 \mathrm{~Hz}, 2 \mathrm{H}$, phenyl-H), $6.75(\mathrm{~d}, J=8.8 \mathrm{~Hz}, 2 \mathrm{H}$, phenyl-H), $2.68\left(\mathrm{~s}, 3 \mathrm{H},-\mathrm{CH}_{3}\right) ;{ }^{13} \mathrm{C}$ NMR (101 MHz, DMSO-d $\left.d_{6}\right) \delta 165.1$, $161.7,157.1,148.8,136.3,132.3,131.9,127.6,126.7\left(\mathrm{dd},{ }^{2} J_{\mathrm{C}-}\right.$ $\mathrm{F}=18.9,7.2 \mathrm{~Hz}), 125.3,124.2\left(\mathrm{q},{ }^{1} J_{\mathrm{C}-\mathrm{F}}=272.7 \mathrm{~Hz}\right), 114.8$, $110.1,17.6 ;{ }^{19} \mathrm{~F}$ NMR (377 MHz, DMSO- $\left.d_{6}\right) \delta-61.4$; HRMS
(ESI) $[\mathrm{M}-\mathrm{H}]^{-}$calcd for $\mathrm{C}_{18} \mathrm{H}_{12} \mathrm{ON}_{3} \mathrm{BrF}_{3} \mathrm{~S}$ : 453.9846, found: 453.9831 .

2.17. 4-Methyl-N',2-bis(4-(trifluoromethyl)phenyl)thiazole-5carbohydrazide (3n). A white solid, m. p. $234.3-235.5^{\circ} \mathrm{C}$, yield 68.4\%; ${ }^{1} \mathrm{H}$ NMR (400 MHz, DMSO-d $) \delta 10.43(\mathrm{~s}, 1 \mathrm{H}$, -CONH-), 8.67 (s, 1H, -NH-), 8.18 (d, J=8.1 Hz, 2H, phenylH), $7.89(\mathrm{~d}, J=8.3 \mathrm{~Hz}, 2 \mathrm{H}$, phenyl-H), $7.51(\mathrm{~d}, J=8.5 \mathrm{~Hz}, 2 \mathrm{H}$, phenyl-H), $6.90(\mathrm{~d}, J=8.5 \mathrm{~Hz}, 2 \mathrm{H}$, phenyl-H), $2.69(\mathrm{~s}, 3 \mathrm{H}$, $\left.-\mathrm{CH}_{3}\right) ;{ }^{13} \mathrm{C}$ NMR $\left(101 \mathrm{MHz}, \mathrm{DMSO}-d_{6}\right) \delta 165.2,161.7,157.4$, $152.6,136.2,131.1\left(\mathrm{q},{ }^{2} J_{\mathrm{C}-\mathrm{F}}=32.3 \mathrm{~Hz}\right), 127.6,126.8,126.1(\mathrm{q}$, $\left.{ }^{1} J_{\mathrm{C}-\mathrm{F}}=271.7 \mathrm{~Hz}\right), 125.1,124.4\left(\mathrm{q},{ }^{1} J_{\mathrm{C}-\mathrm{F}}=272.4 \mathrm{~Hz}\right), 119.1(\mathrm{q}$, $\left.{ }^{2} J_{\mathrm{C}-\mathrm{F}}=31.6 \mathrm{~Hz}\right), 113.2,112.1,17.7 ;{ }^{19} \mathrm{~F}$ NMR $(377 \mathrm{MHz}$, DMSO- $\left.d_{6}\right) \delta-59.4,-61.5$; HRMS (ESI) $[\mathrm{M}-\mathrm{H}]^{-}$calcd for $\mathrm{C}_{19} \mathrm{H}_{12} \mathrm{ON}_{3} \mathrm{~F}_{9} \mathrm{~S}$ : 444.0608, found: 444.0600 .

2.18. $\quad N^{\prime}$-(3-Chlorophenyl)-4-methyl-2-(4-(trifluoromethyl) phenyl)thiazole-5-carbohydrazide (3o). A white solid, m. p. 201.3-202.6 ${ }^{\circ} \mathrm{C}$, yield 44.6\%; ${ }^{1} \mathrm{H}$ NMR $\left(400 \mathrm{MHz}, \mathrm{DMSO}-d_{6}\right)$ $\delta 10.36(\mathrm{~s}, 1 \mathrm{H},-\mathrm{CONH}-), 8.38$ (s, 1H, -NH-), 8.20 (d, $J=8.1 \mathrm{~Hz}, 2 \mathrm{H}$, phenyl-H), 7.90 (d, $J=8.3 \mathrm{~Hz}, 2 \mathrm{H}$, phenyl-H), $7.21(\mathrm{t}, J=7.9 \mathrm{~Hz}, 1 \mathrm{H}$, phenyl-H), 6.86-6.80 (m, $1 \mathrm{H}$, phenyl$\mathrm{H}), 6.79(\mathrm{~d}, J=5.6 \mathrm{~Hz}, 1 \mathrm{H}$, phenyl-H), $6.77(\mathrm{~d}, J=5.3 \mathrm{~Hz}, 1 \mathrm{H}$, phenyl-H), 2.71 (s, 3H, - $\left.\mathrm{CH}_{3}\right) ;{ }^{13} \mathrm{C}$ NMR (101 MHz, DMSO$\left.d_{6}\right) \delta 165.2,161.7,157.2,151.1,136.3,134.0,131.0,127.6$, $127.4,126.8$ (dd, $J=9.5,5.9 \mathrm{~Hz}), 125.2,124.4(\mathrm{dd}, J=544.5$, $272.3 \mathrm{~Hz}), 118.8,112.1,111.4,17.6 ;{ }^{19} \mathrm{~F} \mathrm{NMR}(377 \mathrm{MHz}$, DMSO- $\left.d_{6}\right) \quad \delta$-61.4; HRMS (ESI) $[\mathrm{M}-\mathrm{H}]^{-}$calcd for $\mathrm{C}_{18} \mathrm{H}_{14} \mathrm{ON}_{3} \mathrm{ClF}_{3} \mathrm{~S}$ : 412.0490, found: 412.0492 .

\section{Results and Discussion}

3.1. Synthesis of Target Compounds $\mathbf{3 a - 3 o}$. As shown in Scheme 1, the starting material substituted thiobenzamide was subjected by two sequential reactions including cyclization and hydrolysis to provide a crucial intermediate 2-(4chlorophenyl)-4-methylthiazole-5-carboxylic acid (2a) or 4methyl-2-(4-(trifluoromethyl)phenyl)thiazole-5-carboxylic acid (2b). The final target compounds $\mathbf{3} \mathbf{a}-\mathbf{3} \mathbf{3}$ were obtained through the condensation reaction between $\mathbf{2 a}$ (or $\mathbf{2} \mathbf{b}$ ) and different substituted phenylhydrazines in solvent $\mathrm{CH}_{2} \mathrm{Cl}_{2}$ at $25^{\circ} \mathrm{C}$. These chemical structures were determined by using NMR and HRMS analyses (Figures S1-S61, Supplementary Materials).

3.2. Antifungal Activities of Target Compounds $\mathbf{3 a - 3 o}$ and Structure-Activity Relationship (SAR). Their antifungal activity against aforementioned six kinds of plant pathogens was evaluated by using the mycelium growth rate approach, while the most applied antimicrobial drugs, namely, fluopyram (FP), boscalid (BS), hymexazol (HM), prochloraz (PC), and carbendazim (CB) were co-assayed for comparison. Table 1 reveals that some of the target compounds were identified with excellent antimicrobial activities at $25.0 \mu \mathrm{g} / \mathrm{mL}$. For the biological effect against $B . d$. strain, compounds $\mathbf{3 a}, \mathbf{3 b}, \mathbf{3 d}$, and $\mathbf{3} \mathbf{g}-\mathbf{3 l}$ gave the inhibition rate within $70.0-88.5 \%$, which were better than positive 


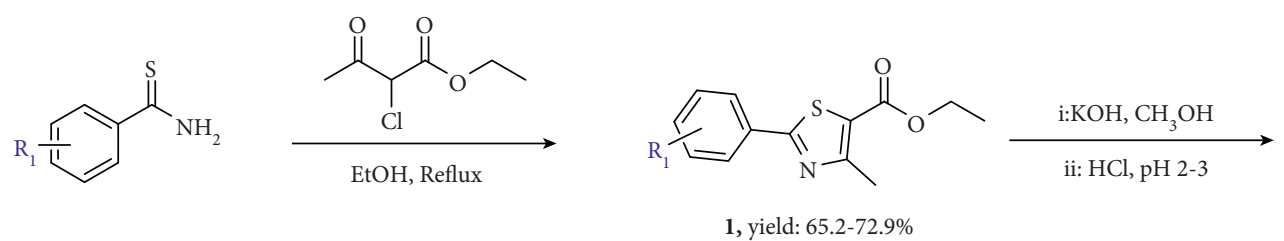

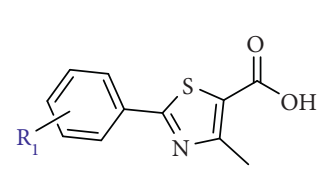

2a-2b, yield: 69.5-78.3\%

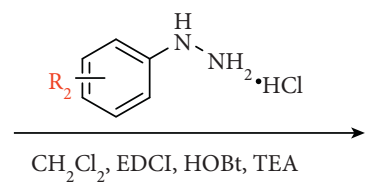

3a: $\mathrm{R}_{1}=4-\mathrm{Cl}, \mathrm{R}_{2}=\mathrm{H}$

3b: $\mathrm{R}_{1}=4-\mathrm{Cl}, \mathrm{R}_{2}=2-\mathrm{F}$

3c: $\mathrm{R}_{1}=4-\mathrm{Cl}, \mathrm{R}_{2}=3-\mathrm{F}$

3d: $\mathrm{R}_{1}=4-\mathrm{Cl}, \mathrm{R}_{2}=4-\mathrm{F}$

3e: $\mathrm{R}_{1}=4-\mathrm{Cl}, \mathrm{R}_{2}=4-\mathrm{Br}$

3f: $\mathrm{R}_{1}=4-\mathrm{Cl}, \mathrm{R}_{2}=4-\mathrm{CF}_{3}$

3g: $\mathrm{R}_{1}=4-\mathrm{Cl}, \mathrm{R}_{2}=3-\mathrm{Cl}^{3}$

$3 \mathrm{~h}: \mathrm{R}_{1}=4-\mathrm{Cl}, \mathrm{R}_{2}=4-\mathrm{Cl}$

$$
\begin{aligned}
& \text { 3i: } \mathrm{R}_{1}=4-\mathrm{CF}_{3}, \mathrm{R}_{2}=\mathrm{H} \\
& \text { 3j: } \mathrm{R}_{1}=4-\mathrm{CF}_{3}, \mathrm{R}_{2}=2-\mathrm{F} \\
& \text { 3k: } \mathrm{R}_{1}=4-\mathrm{CF}_{3}, \mathrm{R}_{2}=3-\mathrm{F} \\
& \text { 31: } \mathrm{R}_{1}=4-\mathrm{CF}_{3}, \mathrm{R}_{2}=4-\mathrm{F} \\
& \text { 3m: } \mathrm{R}_{1}=4-\mathrm{CF}_{3}, \mathrm{R}_{2}=4-\mathrm{Br} \\
& \text { 3n: } \mathrm{R}_{1}=4-\mathrm{CF}_{3}, \mathrm{R}_{2}=4-\mathrm{CF}_{3} \\
& \text { 3o: } \mathrm{R}_{1}=4-\mathrm{CF}_{3}, \mathrm{R}_{2}=3-\mathrm{Cl}
\end{aligned}
$$

\begin{tabular}{|c|c|c|c|c|c|c|}
\hline \multirow{2}{*}{ Compounds } & \multicolumn{6}{|c|}{ Inhibition rate (\%) } \\
\hline & B. $d$. & G. s. & $V . d$. & T. c. & S. $s$. & F. $o$. \\
\hline $3 a$ & $70.0 \pm 1.7$ & $78.7 \pm 0.7$ & $67.7 \pm 0.9$ & $70.0 \pm 1.5$ & $48.0 \pm 1.7$ & $25.7 \pm 0.5$ \\
\hline $3 b$ & $72.3 \pm 1.2$ & $71.8 \pm 0.8$ & $79.7 \pm 0.6$ & $83.6 \pm 1.2$ & $73.9 \pm 0.5$ & $76.1 \pm 0.5$ \\
\hline $3 c$ & $67.8 \pm 1.3$ & $58.5 \pm 0.9$ & $46.7 \pm 0.6$ & $71.8 \pm 0.4$ & $40.3 \pm 1.0$ & $63.7 \pm 0.6$ \\
\hline $3 d$ & $80.2 \pm 1.7$ & $86.1 \pm 1.0$ & $81.1 \pm 0.6$ & $77.7 \pm 0.6$ & $65.8 \pm 0.8$ & $12.8 \pm 1.7$ \\
\hline $3 e$ & $65.0 \pm 1.0$ & $54.4 \pm 0.5$ & $51.5 \pm 1.5$ & $25.7 \pm 0.6$ & $47.2 \pm 2.1$ & 0 \\
\hline $3 f$ & $41.0 \pm 1.0$ & $20.4 \pm 1.4$ & $41.1 \pm 0.5$ & $11.3 \pm 0.5$ & $41.2 \pm 0.9$ & 0 \\
\hline $3 g$ & $76.9 \pm 1.8$ & $64.8 \pm 1.8$ & $34.9 \pm 1.6$ & $49.8 \pm 1.0$ & 0 & $19.0 \pm 1.0$ \\
\hline $3 \mathrm{~h}$ & $88.5 \pm 1.0$ & $69.9 \pm 0.5$ & $68.6 \pm 1.6$ & $68.3 \pm 0.6$ & $44.0 \pm 1.2$ & $6.4 \pm 0.9$ \\
\hline $3 \mathbf{i}$ & $71.5 \pm 0.8$ & $55.3 \pm 0.4$ & $56.4 \pm 1.0$ & $52.0 \pm 1.7$ & $50.0 \pm 1.8$ & $42.8 \pm 1.3$ \\
\hline $3 \mathbf{j}$ & $74.5 \pm 0.9$ & $51.6 \pm 0.1$ & $66.7 \pm 0.7$ & $90.3 \pm 0.6$ & $69.1 \pm 0.1$ & $50.3 \pm 0.9$ \\
\hline $3 \mathbf{k}$ & $74.7 \pm 1.0$ & $71.3 \pm 0.5$ & $56.7 \pm 1.0$ & $98.7 \pm 0.6$ & $85.5 \pm 1.5$ & $55.3 \pm 0.1$ \\
\hline 31 & $79.3 \pm 0.8$ & $80.5 \pm 0.5$ & $80.8 \pm 1.0$ & $81.1 \pm 0.6$ & $72.7 \pm 1.7$ & 0 \\
\hline $3 \mathrm{~m}$ & $65.0 \pm 1.0$ & $54.4 \pm 0.5$ & $51.5 \pm 1.5$ & $25.7 \pm 0.6$ & $47.2 \pm 2.1$ & 0 \\
\hline $3 n$ & $8.0 \pm 1.0$ & $17.3 \pm 2.0$ & $44.6 \pm 1.6$ & $19.7 \pm 1.3$ & $33.8 \pm 0.1$ & 0 \\
\hline 30 & $62.1 \pm 1.1$ & $29.7 \pm 1.3$ & $16.7 \pm 1.4$ & $20.7 \pm 1.8$ & 0 & $32.0 \pm 1.0$ \\
\hline FP & $35.3 \pm 0.6$ & $46.9 \pm 1.3$ & $21.3 \pm 1.2$ & $10.1 \pm 1.5$ & $66.1 \pm 1.0$ & $25.7 \pm 1.0$ \\
\hline BS & $36.5 \pm 1.0$ & $19.8 \pm 1.0$ & $82.7 \pm 2.3$ & $70.6 \pm 1.0$ & $62.8 \pm 1.0$ & $13.4 \pm 1.4$ \\
\hline HM & $15.4 \pm 1.0$ & $44.6 \pm 1.0$ & $30.0 \pm 1.0$ & $75.2 \pm 0.6$ & $26.9 \pm 1.0$ & $50.6 \pm 0.5$ \\
\hline CB & 100 & 100 & $33.0 \pm 0.5$ & 100 & 100 & 100 \\
\hline PC & 100 & 100 & $75.6 \pm 0.5$ & $37.2 \pm 1.2$ & 100 & 100 \\
\hline
\end{tabular}

3a-3o, yield: 13.7-68.4\%

Scheme 1: Synthetic route for target compounds $\mathbf{3 a - 3 o . ~}$

TABLE 1: Inhibition rates of compounds 3a-3o against B. d., G. s., V. d., T. c., S. s., and F. o. at $25.0 \mu \mathrm{g} / \mathrm{mL}$.

drugs FP (35.3\%), BS (36.5\%), and HM (15.4\%), but lower compared to CB (100\%) and PC (100\%). For the anti-G. s. activity, compounds $\mathbf{3} \mathbf{a}, \mathbf{3} \mathbf{b}, \mathbf{3} \mathbf{d}, \mathbf{3} \mathbf{k}$, and $\mathbf{3 l}$ afforded the inhibitory effect of $78.7 \%, 71.8 \%, 86.1 \%, 71.3 \%$, and $80.5 \%$, respectively. For the anti- $V$. $d$. activity, compounds $\mathbf{3 b}, \mathbf{3 d}$, and 31 gave the appreciable bioactivity with ratios of $79.7 \%$, $81.1 \%$, and $80.8 \%$, respectively. By contrast, compound $3 \mathbf{k}$ was extremely bioactive against $T$. c. strain with the inhibition ratio of $98.7 \%$. Simultaneously, this compound (3k) also displayed the best anti-S. $s$. activity with inhibitory rate of $85.5 \%$. For the anti-F. o. activity, compound $\mathbf{3 b}$ yielded the best bioactivity with the rate of $76.1 \%$. Among all the target molecules, compounds $\mathbf{3 b}, \mathbf{3} \mathbf{j}$, and $\mathbf{3 k}$ displayed the comprehensive bioactivity against all the tested fungal strains (B.d., G. s., V.d., T.c., S. s., and F. o.) with the corresponding inhibitory rates of $72.3 \%, 71.8 \%, 79.7 \%$, $83.6 \%, 73.9 \%$, and $76.1 \%$ (for compound $3 \mathbf{b}$ ), $74.5 \%, 51.6 \%$, $66.7 \%, 90.3 \%, 69.1 \%$, and $50.3 \%$ (for compound $3 \mathbf{j}$ ), and $74.7 \%, 71.3 \%, 56.7 \%, 98.7 \%, 85.5 \%$, and $55.3 \%$ (for compound $3 \mathbf{k})$. After careful observation, it was found that the 
TABLE 2: $\mathrm{EC}_{50}$ values of compounds $\mathbf{3 a - 3 0}$ against tested fungi in vitro.

\begin{tabular}{|c|c|c|c|c|c|c|c|}
\hline Comp. & Pathogens & Regression equation & $\mathrm{EC}_{50}(\mu \mathrm{g} / \mathrm{mL})$ & Comp. & Pathogens & Regression equation & $\mathrm{EC}_{50}(\mu \mathrm{g} / \mathrm{mL})$ \\
\hline \multirow{4}{*}{$3 a$} & B. $d$. & $y=0.493 x+4.987$ & $1.05 \pm 0.17$ & \multirow{2}{*}{$3 \mathrm{~h}$} & $V . d$. & $y=0.541 x+4.696$ & $3.63 \pm 0.18$ \\
\hline & G. s. & $y=1.104 x+4.220$ & $5.08 \pm 0.20$ & & T. c. & $y=1.186 x+3.626$ & $14.4 \pm 0.33$ \\
\hline & S. $s$. & $y=1.491 x+3.361$ & $12.6 \pm 0.6$ & \multirow[t]{2}{*}{$3 \mathbf{i}$} & B. $d$. & $y=1.028 x+4.992$ & $1.01 \pm 0.09$ \\
\hline & T. c. & $y=0.759 x+4.110$ & $14.9 \pm 1.3$ & & B. $d$. & $y=0.562 x+4.483$ & $1.75 \pm 0.16$ \\
\hline \multirow{6}{*}{$3 b$} & B. $d$. & $y=0.460 x+4.937$ & $1.36 \pm 0.53$ & \multirow{2}{*}{$3 \mathbf{j}$} & S. $s$. & $y=0.508 x+4.547$ & $7.77 \pm 0.70$ \\
\hline & G. s. & $y=0.476 x+4.977$ & $1.11 \pm 0.13$ & & $V . d$. & $y=0.672 x+4.543$ & $4.78 \pm 0.32$ \\
\hline & S. s. & $y=1.545 x+3.651$ & $7.45 \pm 0.29$ & \multirow{4}{*}{$3 k$} & T. c. & $y=1.726 x+4.269$ & $2.64 \pm 0.06$ \\
\hline & $V . d$. & $y=0.677 x+4.939$ & $1.22 \pm 0.25$ & & B. $d$. & $y=0.476 x+5.030$ & $0.86 \pm 0.12$ \\
\hline & T. c. & $y=0.905 x+4.444$ & $4.10 \pm 0.27$ & & G. s. & $y=0.596 x+4.772$ & $2.41 \pm 0.15$ \\
\hline & F. $o$. & $y=1.162 x+3.984$ & $7.47 \pm 0.21$ & & S. $s$. & $y=1.352 x+3.559$ & $11.6 \pm 0.38$ \\
\hline \multirow{4}{*}{$3 c$} & B. $d$. & $y=0.351 x+4.939$ & $1.48 \pm 0.22$ & \multirow{5}{*}{31} & T. $c$. & $y=1.578 x+4.636$ & $1.70 \pm 0.02$ \\
\hline & G. $s$. & $y=0.281 x+4.806$ & $4.89 \pm 0.52$ & & B. $d$. & $y=0.547 x+5.123$ & $0.59 \pm 0.04$ \\
\hline & T. $c$. & $y=0.827 x+4.219$ & $8.77 \pm 0.85$ & & G. s. & $y=1.163 x+5.186$ & $0.69 \pm 0.04$ \\
\hline & F. $o$. & $y=0.528 x+4.318$ & $19.6 \pm 3.5$ & & S. $s$. & $y=1.352 x+3.559$ & $5.57 \pm 0.30$ \\
\hline \multirow{5}{*}{$3 d$} & B. $d$. & $y=0.585 x+4.883$ & $1.58 \pm 0.06$ & & $V . d$. & $y=1.208 x+4.820$ & $1.40 \pm 0.06$ \\
\hline & G. $s$. & $y=1.048 x+4.721$ & $1.84 \pm 0.12$ & \multirow{4}{*}{$\begin{array}{c}3 \mathrm{~m} \\
30\end{array}$} & T. c. & $y=1.150 x+4.330$ & $3.82 \pm 0.17$ \\
\hline & S. $s$. & $y=2.100 x+2.913$ & $9.48 \pm 0.24$ & & B. $d$. & $y=0.695 x+4.373$ & $7.97 \pm 0.91$ \\
\hline & $V . d$. & $y=1.171 x+4.346$ & $3.61 \pm 0.15$ & & B. $d$. & $y=0.690 x+4.392$ & $7.60 \pm 0.51$ \\
\hline & T. $c$. & $y=0.967 x+4.478$ & $3.46 \pm 0.23$ & & B. $d$. & $y=1.131 x+5.996$ & $0.13 \pm 0.01$ \\
\hline $3 e$ & B. $d$. & $y=0.695 x+4.373$ & $7.97 \pm 0.91$ & \multirow{5}{*}{ CB } & G. s. & $y=4.016 x+5.629$ & $0.70 \pm 0.01$ \\
\hline \multirow{2}{*}{$3 f$} & B. $d$. & $y=0.507 x+5.015$ & $0.93 \pm 0.03$ & & S. $s$. & $y=3.322 x+7.326$ & $0.20 \pm 0.01$ \\
\hline & G. $s$. & $y=0.524 x+4.682$ & $4.05 \pm 0.12$ & & T. $c$. & $y=2.322 x+4.772$ & $1.25 \pm 0.05$ \\
\hline \multirow{2}{*}{$3 g$} & B. $d$. & $y=0.520 x+5.133$ & $0.55 \pm 0.02$ & & F. $o$. & $y=4.116 x+6.058$ & $0.55 \pm 0.01$ \\
\hline & G. s. & $y=0.558 x+4.815$ & $2.14 \pm 0.21$ & & & & \\
\hline
\end{tabular}

final bioactivity of target compounds could be significantly affected by a number of factors, such as the kind and electronic property of substitutional group, the type of halogen, and the location of halogen on the benzene ring. The detailed SAR is summarized as follows. (1) As $R_{1}=4$ $\mathrm{Cl}$, introducing $2-\mathrm{F}(3 \mathbf{b})$ and $4-\mathrm{F}(3 \mathbf{d})$ on the benzene ring could improve the bioactivity against B. d., G. s., V. d., T. c., and $S$. s. strains compared to $3 \mathbf{a}\left(R_{2}=\mathrm{H}\right)$, while $R_{2}=3-\mathrm{F}$ and 4 - $\mathrm{Br}$ normally gave a reduced tendency on the bioactivity. (2) A strong electron-withdrawing group $\left(R_{2}=4-\mathrm{CF}_{3}, 3 \mathbf{3 f}\right)$ significantly decreased the antifungal activity. (3) The halogen atom at the para-position showed better bioactivity than that of meta-position, illustrated by compounds $3 \mathbf{d}$ (4F, $80.2 \%, 86.1 \%, 81.1 \%, 77.7 \%$, and $65.8 \%$ against $B$. d., G. s., V.d., T. c., and S. s., respectively) and 3c (3-F, $67.8 \%, 58.5 \%$, $46.7 \%, 71.8 \%$, and $40.3 \%$ against B. d., G. s., V. d., T. c., and S. s., respectively), and $3 \mathbf{h}(4-\mathrm{Cl}, 88.5 \%, 69.9 \%, 68.6 \%$, $68.3 \%$, and $44.0 \%$ against B. d., G. s., V. d., T. c., and S. s., respectively) and $\mathbf{3 g}(3-\mathrm{Cl}, 76.9 \%, 64.8 \%, 34.9 \%, 49.8 \%$, and 0 against $B$. d., G. s., $V . d$. ., T. c., and S. s., respectively). (4) As $R_{1}=4-\mathrm{CF}_{3}$, introducing $2-\mathrm{F}, 3-\mathrm{F}$, or $4-\mathrm{F}$ could distinctly enhance the anti-T. c. activity from $52.0 \%(-\mathrm{H}, 3 \mathbf{i})$ to $90.3 \%$ (2-F, 3j), 98.7\% (3-F, 3k), and 81.1\% (4-F, 31), respectively. (5) Similarly, the strong electron-withdrawing group $\left(R_{2}=4-\mathrm{CF}_{3}, 3 \mathbf{n}\right)$ significantly reduced the antifungal activity. (6) The bioactivity comparison of $\mathbf{3 a}-\mathbf{3} \mathbf{g}\left(R_{1}=4-\mathrm{Cl}\right)$ and $3 \mathbf{i}-3 \mathbf{o}\left(R_{1}=4-\mathrm{CF}_{3}\right)$ revealed that the former series compound normally gave a relatively ameliorative antifungal activity.

Further bioactivity screening results are shown in Table 2 and expressed by $\mathrm{EC}_{50}$ values. Clearly, compounds $\mathbf{3 g}, \mathbf{3 h}, \mathbf{3 k}$, and $\mathbf{3 l}$ provided excellent biological actions against $B$. $d$., with the corresponding values of $0.93,0.55,0.86$, and $0.59 \mu \mathrm{g} / \mathrm{mL}$.
The $\mathrm{EC}_{50}$ values of these compounds were close to $\mathrm{CB}$ $(0.13 \mu \mathrm{g} / \mathrm{mL})$. Compounds $\mathbf{3 b}, \mathbf{3 d}, \mathbf{3 h}, \mathbf{3 k}$, and $\mathbf{3 l}$ showed good anti-G. s. activity with $\mathrm{EC}_{50}$ values of $1.11,1.84,2.14,2.41$, and $0.69 \mu \mathrm{g} / \mathrm{mL}$, respectively. Distinctly, compound 31 exerted the comparative inhibitory effect than that of CB $(0.70 \mu \mathrm{g} / \mathrm{mL})$. Compounds $\mathbf{3 b}, \mathbf{3 d}, \mathbf{3 h}, \mathbf{3} \mathbf{j}$, and $\mathbf{3 1}$ were bioactive against $V$. $d$. with the corresponding $\mathrm{EC}_{50}$ values of 1.22, 3.61, 3.63, 4.78, and $1.40 \mu \mathrm{g} / \mathrm{mL}$. By contrast, compounds $\mathbf{3 a}, \mathbf{3 b}, \mathbf{3 d}, \mathbf{3 j}$, and $3 \mathbf{k}$ provided moderate anti-S. $s$. activity with $\mathrm{EC}_{50}$ values of $12.6,7.45,9.48,7.77$, and $11.6 \mu \mathrm{g} / \mathrm{mL}$, respectively. For the anti-T. c. activity, compounds $\mathbf{3} \mathbf{b}, \mathbf{3} \mathbf{d}, \mathbf{3} \mathbf{j}, \mathbf{3 k}$, and $\mathbf{3} \mathbf{l}$ showed good inhibitory effect with $\mathrm{EC}_{50}$ values of $4.10,3.46,2.64,1.70$, and $3.82 \mu \mathrm{g} / \mathrm{mL}$, respectively. By contrast, only compounds $\mathbf{3 b}$ and $3 \mathrm{c}$ had good $\mathrm{EC}_{50}$ values against $V . d$. (7.47 and $19.6 \mu \mathrm{g} /$ $\mathrm{mL}$, respectively). The above result indicated that the rational design of thiazolyl hydrazine derivatives can help in acquiring high bioactive substrates.

\section{Conclusions}

In summary, novel simple thiazolyl hydrazide derivatives were prepared and assessed for their antifungal activities. The result indicated that most of these designed compounds gave good antifungal activity against the tested six fungi. Compounds $\mathbf{3 a}, \mathbf{3 b}, \mathbf{3 c}, \mathbf{3 d}$, and $\mathbf{3 g}-\mathbf{3 o}$ displayed strong bioactivity against $B$. d., providing $\mathrm{EC}_{50}$ values ranging from 0.59 to $1.75 \mu \mathrm{g} / \mathrm{mL}$, which were better than controlled drugs of fluopyram $(>25 \mu \mathrm{g} / \mathrm{mL})$, hymexazol $(>25 \mu \mathrm{g} / \mathrm{mL})$, and boscalid $(>25 \mu \mathrm{g} / \mathrm{mL})$ and slightly weaker than carbendazim $(0.13 \mu \mathrm{g} / \mathrm{mL})$. Compounds $\mathbf{3 b}, \mathbf{3 d}$, and $\mathbf{3 l}$ displayed excellent bioactivity against $G$. $s$. with $\mathrm{EC}_{50}$ values of $1.11,1.84$, and $0.69 \mu \mathrm{g} / \mathrm{mL}$, respectively. Given these excellent bioactivities, 
this novel thiazolyl hydrazide framework can provide a good starting point for designing more potent antifungal agents.

\section{Data Availability}

The data supporting these results are available from the corresponding author upon request.

\section{Conflicts of Interest}

The authors declare that they have no conflicts of interest.

\section{Authors' Contributions}

Jianjun Zhu was responsible for investigation, data curation, formal analysis, methodology, and original draft preparation. Yazhen Chen and Fen Su were responsible for data curation, formal analysis, and methodology. Peiyi Wang was responsible for data curation, supervision, formal analysis, and review and editing.

\section{Acknowledgments}

We acknowledge the financial support provided by the National Natural Science Foundation of China (31860516, 21662009, and 21702037).

\section{Supplementary Materials}

The supplementary data contain the related NMR and HRMS spectra for the intermediates $\mathbf{2} \mathbf{a}-\mathbf{2} \mathbf{b}$ and target compounds 3a-3o (Figures S1-S61). (Supplementary Materials)

\section{References}

[1] H. Sachdeva, R. Saroj, S. Khaturia, D. Dwivedi, and O. P. Chauhan, "Green route for efficient synthesis of novel amino acid schiff bases as potent antibacterial and antifungal agents and evaluation of cytotoxic effects," Journal of Chemistry, vol. 2014, Article ID 848543, 12 pages, 2014.

[2] M. Gupta, S. Sihag, A. K. Varshney, and S. Varshney, "Synthesis, structural, and antimicrobial studies of some new coordination compounds of palladium (II) with azomethines derived from amino acids," Journal of Chemistry, vol. 2013, Article ID 745101, 8 pages, 2013.

[3] R. Gonzalez-Fernandez and J. V. Jorrin-Novo, "Contribution of proteomics to the study of plant pathogenic fungi," Journal of Proteome Research, vol. 11, no. 1, pp. 3-16, 2012.

[4] C. Xu, M. Li, Z. Zhou et al., "Impact of five succinate dehydrogenase inhibitors on DON biosynthesis of Fusarium asiaticum, causing fusarium head blight in wheat," Toxins, vol. 11, no. 5, p. 272, 2019.

[5] A. K. Passari, P. C. Lalsiamthari, L. V. V. Zothanpuia et al., "Biocontrol of fusarium wilt of capsicum annuum by rhizospheric bacteria isolated from turmeric endowed with plant growth promotion and disease suppression potential," $E$ ropean Journal of Plant Pathology, vol. 150, no. 4, pp. 831-846, 2018.

[6] C. Chen, J. Wang, Q. Luo, S. Yuan, and M. Zhou, "Characterization and fitness of carbendazim-resistant strains of
Fusarium graminearum (wheat scab)," Pest Management Science, vol. 63, no. 12, pp. 1201-1207, 2007.

[7] T. D. Xuan, A. A. Elzaawely, M. Fukuta, and S. Tawata, "Herbicidal and fungicidal activities of lactones in kava (piper methysticum)," Journal of Agricultural and Food Chemistry, vol. 54, no. 3, pp. 720-725, 2006.

[8] L. Wang, C. Li, Y. Zhang, C. Qiao, and Y. Ye, "Synthesis and biological evaluation of benzofuroxan derivatives as fungicides against phytopathogenic fungi," Journal of Agricultural and Food Chemistry, vol. 61, no. 36, pp. 8632-8640, 2013.

[9] A. D. Ribas e Ribas, P. Spolti, E. M. Del Ponte, K. Z. Donato, H. Schrekker, and A. M. Fuentefria, "Is the emergence of fungal resistance to medical triazoles related to their use in the agroecosystems? a mini review," Brazilian Journal of Microbiology, vol. 47, no. 4, pp. 793-799, 2016.

[10] Q.-F. Wu, B. Zhao, Z.-J. Fan et al., "Design, synthesis and fungicidal activity of isothiazole-thiazole derivatives," $R S C$ Advances, vol. 8, no. 69, pp. 39593-39601, 2018.

[11] X. Zuo, N. Mi, Z. Fan et al., "Synthesis of 4-methyl-1, 2, 3thiadiazole derivatives via ugi reaction and their biological activities," Journal of Agricultural and Food Chemistry, vol. 58, no. 5, pp. 2755-2762, 2010.

[12] W. T. Mao, H. Zhao, Z. J. Fan et al., "Synthesis and bioactivity of N-tertbutyl-N'-acyl-5-methyl-1, 2, 3-thiadiazole-4-carbohydrazides," Chinese Chemical Letters, vol. 23, no. 11, pp. p1233-1236, 2012.

[13] M. Wu, Q. Sun, C. Yang et al., "Synthesis and activity of combretastatin A-4 analogues: 1, 2, 3-thiadiazoles as potent antitumor agents," Bioorganic \& Medicinal Chemistry Letters, vol. 17, no. 4, pp. 869-873, 2007.

[14] X. H. Gu, X. Z. Wan, and B. Jiang, "Syntheses and biological activities of bis (3-indolyl) thiazoles, analogues of marine bis (indole) alkaloid nortopsentins," Bioorganic \& Medicinal Chemistry Letters, vol. 9, no. 4, pp. 569-572, 1999.

[15] V. Spano, A. Attanzio, S. Cascioferro et al., "Synthesis and antitumor activity of new thiazole nortopsentin analogs," Marine Drugs, vol. 14, no. 12, p. p226, 2016.

[16] D. Kumar, N. M. Kumar, K. H. Chang, R. Gupta, and K. Shah, "Synthesis and in-vitro anticancer activity of 3, 5-bis (indolyl)-1, 2, 4-thiadiazoles," Bioorganic \& Medicinal Chemistry Letters, vol. 21, no. 19, pp. 5897-5900, 2011.

[17] D.-S. Kim, S.-J. Chun, J.-J. Jeon, S.-W. Lee, and G.-H. Joe, "Synthesis and fungicidal activity of ethaboxam against oomycetes," Pest Management Science, vol. 60, no. 10, pp. 1007-1012, 2004.

[18] Y.-J. Zhang, J. Li, W. Zhao, and M.-G. Zhou, "A single amino acid substitution in the $S \mathrm{dhB}$ protein of succinate dehydrogenase determines resistance to amicarthiazol in Xanthomonas oryzae pv. oryzae," Pest Management Science, vol. 66, no. 6, pp. 627-633, 2010.

[19] N. Matuszak, B. Es Saadi, G. Labar, J. Marchand-Brynaert, and D. M. Lambert, "Benzisothiazolinone as a useful template for the design of new monoacylglycerol lipase inhibitors: investigation of the target residues and comparison with octhilinone," Bioorganic \& Medicinal Chemistry Letters, vol. 21, no. 24, pp. 7321-7324, 2011.

[20] R. J. Fussell, K. Jackson Addie, S. L. Reynolds, and M. F. Wilson, "Assessment of the stability of pesticides during cryogenic sample processing. 1. apples," Journal of Agricultural and Food Chemistry, vol. 50, no. 3, pp. 441-448, 2002.

[21] J. Shang, R. F. Sun, Y. Q. Li, R. Q. Huang, F. C. Bi, and Q. M. Wang, "Synthesis and insecticidal evaluation of N-tertbutyl-N'-thio [1-(6-chloro-3-pyridylmethyl)-2-nitroiminoimidazolidine]-N, $\mathrm{N}^{\prime}$-diacylhydrazines," Journal of 
Agricultural and Food Chemistry, vol. 58, no. 3, pp. p1834-1837, 2010.

[22] Y. Liu, H. Song, Y. Huang et al., "Design, synthesis, and antiviral, fungicidal, and insecticidal activities of tetrahydro$\beta$-carboline-3-carbohydrazide derivatives," Journal of Agricultural and Food Chemistry, vol. 62, no. 41, pp. 9987-9999, 2014.

[23] I. Travlos, E. Rapti, I. Gazoulis et al., "The herbicidal potential of different pelargonic acid products and essential oils against several important weed species," Agronomy-Basel, vol. 10, no. 11, p. 1687, 2020.

[24] J. E. Sheridan and V. Vachrabhorn, "Mercury-resistant Pyrenophora aveniiein new Zealand seed oats: a comparison of six different compounds as seed disinfectants," New Zealand Journal of Agricultural Research, vol. 11, no. 4, pp. 812-815, 1968.

[25] C. Bicchi, C. Cordero, P. Rubiolo, and A. Occelli, "Determination of daminozide residues in apple pulp using HPLC-DAD-UV," Journal of Agricultural and Food Chemistry, vol. 49, no. 8, pp. 3548-3552, 2001.

[26] P.-L. Zhao, J. Li, and G.-F. Yang, "Synthesis and insecticidal activity of chromanone and chromone analogues of diacylhydrazines," Bioorganic \& Medicinal Chemistry, vol. 15, no. 5, pp. 1888-1895, 2007.

[27] T. Tetsuya, Y. Kentaro, and E. Yasuyuki, "Cyclic dibenzoylhydrazines reproducing the conformation of ecdysone agonists, RH-5849," Bioorganic \& Medicinal Chemistry, vol. 10, no. 4, pp. 953-961, 2002.

[28] P. Y. Wang, L. Zhou, J. Zhou et al., "Synthesis and antibacterial activity of pyridinium-tailored 2, 5-substituted-1, 3, 4-oxadiazole thioether/sulfoxide/sulfone derivatives," Bioorganic \& Medicinal Chemistry Letters, vol. 26, no. 4, pp. 1214-1217, 2016.

[29] Q.-Q. Tao, L.-W. Liu, P.-Y. Wang et al., "Synthesis and in vitro and in vivo biological activity evaluation and quantitative proteome profiling of oxadiazoles bearing flexible heterocyclic patterns," Journal of Agricultural and Food Chemistry, vol. 67, no. 27, pp. 7626-7639, 2019.

[30] Y. Y. Wu, W. B. Shao, J. J. Zhu et al., "Novel 1, 3, 4-oxadiazole2-carbohydrazides as prospective agricultural antifungal agents potentially targeting succinate dehydrogenase," Journal of Agricultural and Food Chemistry, vol. 67, no. 50, pp. 13892-13903, 2019. 